\title{
Tarda antichità anacronica. Tra storiografia e panegirico
}

\begin{abstract}
This article explores late antique literary culture by making use of a theoretical paradigm recently formulated by the art historians A. Nagel and C. Wood in their book Anachronic Renaissance (New York 2010). Though the period treated in the book, the Renaissance, is very different from late antiquity and though it concentrates on artistic productions, in my view the concept of anachronicity (which is not the same as anachronism) may be applied with profit to the study of late antique texts. In particular, certain key concepts such as the replaceability of the artistic or literary work, imitation of works by themselves, and belatedness, which every work and text inevitably bears in itself, can illuminate some of the aspects of late antique literary production that are most interesting, but which are generally neglected or treated in a superficial way precisely because they are regarded as either secondary or not at all original. These concepts can offer a lens through which to read virtually any late antique text, but here we concentrate on just two works, the Res Gestae of Ammianus Marcellinus and the corpus of the Panegyrici Latini, for one reason above all: both belong to literary genres, historiography and panegyric, which maintain a relation with the past that can be defined as structural. While this is obvious in historiography, in the Panegyrici the sacred value of the past is actualized and projected onto the present time, of which the laudandus, that is, the emperor, is the perfect expression. At the same time, the past itself, vetustas, is presented in the guise of myth, thus undergoing a process of fictionalization which bears elements that destabilize the historiographic discourse, with which the genre of panegyric enters into open competition. In the work of Ammianus, which occupies a unique position within Latin historiography, traces of this same discourse can be identified, indicating a crisis in historiographical representation. The paradigm of anachronicity throws new light on the close relation of the late antique text to the literary tradition of the past, which, it is true, has already been widely investigated, but anachronicity makes it one of its most original and surprising traits. Anachronicity reestablishes the value of belatedness in such a way that the very definition of 'late antiquity' takes on a new meaning, precisely because 'late' is not limited to defining its chronology, but rather assigns it a value that is fundamentally aesthetic.
\end{abstract}

\section{Premessa}

Parlare del concetto di antichità e delle sue rappresentazioni in età tardoantica implica discutere l'essenza stessa di quell'età che noi, impropriamente, chiamiamo 'tarda' antichità (Spätantike, antiquité tardive, late antiquity). La denominazione data 
all'epoca compresa grosso modo tra il III e il VII secolo e avallata dalla storiografia a partire dagli inizi del ventesimo secolo continua nei fatti a suscitare grandi perplessità: l'aggettivo 'tardo', checché se ne dica, non risuona come un neutro indicatore temporale, in quanto inevitabilmente crea una gerarchia di valore tra un'antichità vera e un suo surrogato, un'antichità tarda, e quindi minus habens. Ma anche ammesso che l'aggettivo voglia solo determinare un ovvio fatto cronologico, esso è a parer mio fallace in quanto instaura una consequenzialità che non è solo cronologica ma anche e soprattutto concettuale. La tarda antichità diviene così un logico sviluppo dell'antichità che precede, continuità che viene sancita anche dal fatto che i classicisti detengono una sorta di monopolio dello studio della cultura e in particolare dei testi tardoantichi; ed essi, pervicacemente proprio perché anche inconsciamente, tendono a sottolineare tale continuità piuttosto che metterla in discussione. Ora, se da un lato è necessario comprendere la tarda antichità (termine che si continuerà ad utilizzare faute de mieux) in its own terms, cioè nei suoi tratti distintivi e unici che la rendono diversa dall'antichità, puntando pertanto sugli elementi di rottura piuttosto che su una continuità ricercata a tutti i costi, dall'altro è possibile e necessario ricucire quella rottura e ricercare la continuità partendo da altri presupposti. Il tema della presente raccolta, le rappresentazioni dell'antichità nell'antichità greca e romana, si presta in modo particolarmente adeguato a dare almeno inizio a questo tipo di indagine.

Nelle pagine che seguono intendo seguire una doppia strategia, indicare cioè la rottura e al contempo recuperare la continuità, sebbene da una prospettiva diversa rispetto a quella adottata nella maggior parte dei casi, prendendo abbrivio proprio dalla specifica percezione dell'antichità in alcuni testi latini di età tardoantica. Il fine sarà quello di spiegare perché, tutto sommato, servirsi del termine tarda antichità continua ad avere senso, a patto che l'aggettivo 'tardo' venga investito di una nuova valenza estetica, e non più unicamente cronologica. Nella prima parte introduco il concetto dell'anacronicità, di cui mi servo per spiegare come un diverso approccio che tenga costantemente presente l'alterità della testualità tardoantica rispetto a quella prodotta nelle epoche precedenti sia praticabile insistendo proprio su una percezione del tempo storico fondamentalmente diversa. Nella seconda parte mi limito a citare alcuni esempi tratti da Ammiano Marcellino e dal corpus dei Panegyrici Latini senza alcuna volontà di essere esaustivo né, tanto meno, di volere ripercorrere le varie tappe del discorso critico sui singoli autori. Come spero diverrà chiaro, questo saggio ha un valore euristico, non vuole esibire certezze ma, più modestamente, indicare una via percorribile per chi desidera raggiungere una comprensione più sfumata di una delle epoche più sfuggenti della storia della letteratura occidentale. ${ }^{1}$

1 A fare da background alle riflessioni qui esposte sono alcuni altri miei lavori (si vedano soprattutto Formisano 2007, 2012 e 2014) a cui rimando per una bibliografia più dettagliata. 


\section{Tarda antichità anacronica}

A differenza che in alcuni dei contributi raccolti nel presente volume, qui non ci si pone il compito di individuare e definire cosa intendano gli autori tardoantichi quando ricorrono a termini quali antiquitas o vetustas. Per questo tipo di indagine si può fare riferimento all'utile monografia di Ulrich Eigler, lectiones vetustatis (2003), dedicata alle rappresentazioni dell'antichità nella tarda antichità. In particolare, Eigler si sofferma sulla percezione, diffusa in età tardoantica, della storia romana repubblicana (“die ältere Römische Geschichte”) e della storia imperiale, che col passare del tempo tende a sostuirla in termini di esemplarità. Qui intendo piuttosto concentrarmi sulla percezione del passato in generale, quindi non nei termini di una antiquitas specificamente romana o greca. La prima tappa del percorso ci confronta con un dato di fatto non poi così sorprendente: riflettere sulla percezione del passato implica trattare non solo il tempo della storia, ma anche e sempre quello del presente stesso. Giorgio Agamben, per esempio, ha scritto sul concetto di contemporaneo pagine illuminanti, nelle quali si mette in evidenza proprio l'inevitabilità del rapporto tra presente e passato. Secondo Agamben la contemporaneità è definibile in più modi, tra di essi connessi. Innanzitutto, essa è data da un rapporto discronico con il presente. “Appartiene veramente al suo tempo, è veramente contemporaneo egli scrive - colui che non coincide perfettamente con esso né si adegua alle sue pretese, ed è perciò, in questo senso, inattuale". E ancora, "La contemporaneità è una singolare relazione con il proprio tempo, che aderisce ad esso e, insieme, ne prende le distanze; più precisamente essa è quella relazione col tempo che aderisce ad esso attraverso una sfasatura e un anacronismo". ${ }^{2}$ Ma è soprattutto il particolare rapporto del contemporaneo con il passato che dovrebbe guidare qualsiasi riflessione sulle rappresentazioni dell'antichità. Agamben scrive:

La contemporaneità si iscrive nel presente segnandolo innanzitutto come arcaico e solo chi percepisce nel più moderno e recente gli indici e le segnature dell'arcaico può esserne contemporaneo. Arcaico significa: prossimo all'arché, cioè all'origine. Ma l'origine non è situata soltanto in un passato cronologico: essa è contemporanea al divenire storico e non cessa di operare in questo .... Gli storici della letteratura e dell'arte sanno che fra l'arcaico e il moderno c'è un appuntamento segreto, e non tanto perché le forme più arcaiche sembrano esercitare sul presente un fascino particolare, quanto perché la chiave del moderno è nascosta nell'immemoriale e nel preistorico. Così il mondo antico alla sua fine si volge, per ritrovarsi, ai primordi; l'avanguardia, che si è smarrita nel tempo, insegue il primitivo e l'arcaico. ${ }^{3}$

Ciò che per noi è particolarmente significativo delle dense pagine di Agamben è lo strettissimo rapporto che egli individua tra la percezione del presente e il risorgere dell'‘arcaico' inteso come un'origine non necessariamente relegata ad un tempo

2 Agamben (2009) 20-21 (corsivo nel testo).

3 Ibid., 28-29 (corsivo mio). 
passato ma come Ursprung, che nella definizione di Walter Benjamin (la cui eredità vibra costantemente nelle affermazioni di Agamben, pur non essendo mai nominato nel saggio in questione) designa il momento originario, il primo manifestarsi di un evento o concetto, a differenza di Entstehung, che invece indica la formazione di qualcosa da qualcos'altro e sottolinea pertanto la continuità (le Entstehungen) piuttosto che la rottura o lo stacco improvviso (lo Ursprung). La tendenza della storiografia letteraria, in particolare nella filologia classica, è di privilegiare la continuità, mentre i momenti di rottura da cui una novità si manifesta senza necessariamente essere connessa al passato vengono marginalizzati, forse proprio perché la loro analisi richiederebbe non solo un linguaggio critico diverso da quello normalmente praticato all'interno della disciplina, ma anche a causa del concetto di temporalità non consequenziale richiesto da questo tipo di indagine e che è in contrasto con il lavoro di ricostruzione ritenuta l'unica via percorribile dai più. Seguendo il ragionamento di Agamben, arriviamo alla seconda tappa del nostro breve percorso. Le avanguardie, egli scrive, prediligono l'arcaico: un'affermazione, questa, che potrebbe di primo acchito sembrare paradossale, ma che corrisponde pienamente alle tendenze delle avanguardie storiche (si pensi al primitivismo). Come è noto, negli ultimi decenni alcuni tentativi sono stati fatti per associare lo sperimentalismo formale di certa poesia tardoantica alle tendenze delle avanguardie e del postmoderno. ${ }^{4} \mathrm{Al}$ di là di alcune contraddizioni nel metodo, si tratta di una linea di ricerca importante, soprattutto in quanto instilla il sospetto che i metodi normalmente applicati dall'inteprete classicista possano non essere veramente i più adeguati a comprendere i mutamenti radicali della testualità tardoantica. Ma assimilare questa alle avanguardie richiede un discorso concettualmente più coraggioso, che non si limiti cioè agli aspetti puramente formali del testo, ma che riesca a mettere in luce nuovi risvolti concettuali. Un'avanguardia, infatti, non si definisce unicamente per lo sperimentalismo formale ma anche per una concezione radicalmente diversa del testo e della storia. Un'avanguardia degna di questo nome ha l'urgenza di rompere con il passato in modo tale da fare emergere altre costellazioni concettuali che mettano in crisi non tanto un certo modo di esprimersi quanto i principi stessi della letteratura, dell'arte e della storia. ${ }^{5}$ Se quindi affiniamo quanto è stato affermato sul tardoantico come avanguardia, possiamo approdare ad una considerazione della letteratura del periodo non solo come formalmente diversa da quella antica, ma capace soprattutto di mettere in crisi i paradigmi estetici e concettuali delle età precedenti. La letteratura tardoantica apparirà quindi non più come ossequiosa

4 Lo studio che ha inaugurato questa tendenza è Nugent (1990). Bažil (2013) concentra la propria analisi sull'occorrenza del termine textus (37 volte in tutto) in Ammiano Marcellino, argomentando a favore dell'emergenza del significato odierno del termine in età tardoantica, e concludendo che proprio Ammiano lo pratica in un senso affine alla discussione postmoderna come avviata da Julia Kristeva a proposito di Bakhtin.

5 Rimando alle illuminanti pagine di Didi-Huberman (2007), in particolare il capitolo su Carl Einstein, $149-216$. 
continuatrice ma piuttosto come portatrice di una testualità innovativa che trae la sua linfa vitale proprio dal confronto con la tradizione e con l'antiquitas. In un recente contributo Mark Vessey ci ha ricordato come in realtà l'età tardoantica già in alcuni importanti studi del secolo scorso sia stata percepita da varie prospettive come una sorta di laboratorio che ha prodotto un nuovo concetto di 'letteratura', termine, come si sa, dall'utilizzazione controversa in contesti premoderni. Reinhart Herzog, per esempio, nella sua introduzione al quinto volume dello Handbuch der lateinischen Literatur der Antike (1989), il primo (e l'unico in effetti prodotto) dell'originaria serie di quattro volumi dedicati alla tarda antichità, descrive la letteratura tardoantica come "die erste lateinische, die erste nachrömische Literatur Europas". ${ }^{6}$ Inoltre egli coglie un aspetto essenziale: la letteratura tardoantica cambia radicalmente il senso del testo, che passa dalla rappresentazione all'intepretazione. Nelle parole di Vessey, la mimesi antica si trasforma in ermeneutica tardoantica. ${ }^{7}$ Jacques Derrida, da un lato insiste sulla latinità del termine stesso 'letteratura' rinviando alle sue origini specificamente latine e cristiane, ponendo una questione che meriterebbe di essere discussa più ampiamente di quanto non sia possibile in questa sede:

Existe-t-il, au sens strict et littéral de ce mot (scil. littérature), quelque chose comme de la littérature, comme une institution de la littérature en culture non latino-chrétienne, et plus généralement, bien que les choses soient ici indissociables dans leur histoire, non européenne? Rien n'est moins sure. ${ }^{8}$

Dall'altro lato, nel discutere le Confessiones agostiniane, Derrida mette in evidenza come la condizione della letteratura sia 'passione', cioè la passività del concetto stesso di letteratura, che non può esistere in quanto tale e che può dire tutto e niente (tout dire) visto che essa riceve valore e significato dall'esterno e dalle varie possibili infinite letture. Un testo può essere al contempo testimonianza e finzione, verità e bugia; per questo motivo il termine passione utilizzato da Derrida implica un rapporto strettissimo con il formarsi di una testualità cristiana in età tardoantica. ${ }^{9}$ Come si vedrà nella seconda parte di questo saggio, una delle caratteristiche dei testi tardoantichi è proprio quella di utilizzare il passato, interpretandolo in modo originale, al fine di creare una propria nuova identità: massima innovazione pur nella massima adesione al passato.

Nella terza e ultima tappa del percorso qui seguito si cerca di indagare da una prospettiva diversa il difficile rapporto tra innovazione e tradizione generato dalla cultura e letteratura tardoantica. Come nelle tappe precedenti, anche in questo caso lo stimolo ad accostarsi al fenomeno in modo originale proviene da un campo di

\footnotetext{
6 Herzog (1989) § 18,33,1.

7 Vessey (2015) 24.

8 Derrida (1998) 21 (citato da Vessey 2015, 20).

9 Vessey (2015) 27.
} 
studi diverso dall'antichistica. Prima di entrare nel merito, è necessario chiarire un punto. La teoria dell'anacronicità, che viene qui introdotta, ci può aiutare a cogliere meccanismi letterari molto importanti, che sono già, benché parzialmente, indagati negli studi attuali sul periodo. Ma essa offre un nome e una cornice adeguata per comprendere meglio la letteratura e la cultura tardoantica, e ha anche il grande pregio di metterla in prospettiva storica, di inserire cioè questa epoca, così spesso messa come tra parentesi e liquidata con poche frasi, nella grand narrative della storiografia letteraria e culturale occidentale assegnandole un suo preciso statuto. Per anticipare parte delle conclusioni, la segnatura di questa epoca (per utilizzare un altro termine del pensiero di Agamben) consiste proprio nella sospensione temporale e, come vedremo, nella sua fondamentale astoricità, Geschichtslosigkeit.

In Anachronic Renaissance (2010) gli storici dell'arte americani Alexander Nagel e Christopher Wood introducono un paradigma teorico per approcciare gran parte dell'arte premoderna, sino al Rinascimento. Il primo capitolo, che funge da introduzione, si intitola "La temporalità molteplice dell'opera d'arte" (Plural Temporality of the Work of Art) e si apre con una considerazione tratta da La longue marche di Simone de Beauvoir (un saggio del 1957), in cui l'autrice riflette sulla sua esperienza di visitatrice della città proibita a Pechino: "Il Palazzo Imperiale - afferma Beauvoir - non sembra essere stato restaurato, né tanto meno sembra antico: questa indecisione (cioè tra un aspetto e l'altro) non lo fa sembrare eterno bensì precario e come un'imitazione di se stesso". Proseguendo il suo ragionamento, Beauvoir afferma: "le tracce lasciate dal passato su questo edificio sono così poche che paradossalmente esiterei a chiamarlo un monumento storico" (p. 7). Gli autori commentano le impressioni dell'intellettuale francese facendo riferimento alla tradizione architettonica europea, che costituisce il sostrato delle osservazioni della scrittrice.

Essi affermano: "la storicità di un qualsiasi artefatto è al contempo la fonte della sua autorità e la base per eventuali mistificazioni di quella stessa autorità” (p. 7). Nella tradizione culturale europea moderna, infatti, la storicità è percepita in modo duplice: da un lato essa rende un'opera venerabile proprio in virtù del suo lungo passato, ma dall'altro la stessa opera viene come sminuita allorquando contestualizzata, cioè quando la si approccia dal punto di vista del contesto storico in cui essa è stata immaginata e prodotta: dopo tutto - si pensa - sono stati uomini ad averla fatta, non giganti né déi. Quando Beauvoir osserva stupita il Palazzo Imperiale, tale stupore proviene proprio dalla costatazione che esso non esibisce la propria storia, il proprio lungo passato, non ha nessuna traccia della sua antichità e in quanto tale sembra inautentico, "un'imitazione di se stesso". Come il Palazzo Imperiale di Pechino così tante altre opere risalenti all'età pre-moderna resistono a essere ancorate ad un tempo preciso, cioè a esibire la loro storicità: fissare certe opere (io aggiungerei testi) in un tempo preciso significherebbe ridurle a dimensioni umane, che farebbero dileguare la sacralità su cui esse si fondano. Cogliere la struttura di un determinato oggetto o testo significa astrarsi dal mero oggetto o testo come esso si dà ai sensi: "l’identità di un tale oggetto - affermano Nagel e Wood - è mantenuta, attraverso il tempo, dalla stabilità del nome, che l'opera ha ricevuto, ma anche dalla tacita sostituzione delle sue singole 
parti” (p. 8). Come ha dimostrato Denis Feeney, i romani per esempio non avevano una nozione lineare del tempo e quindi nemmeno una concezione precisa delle date. Essi tendevano invece a rintracciare una miriade di correlazioni tra eventi accaduti o persone vissute in tempi diversi, prescindendo cioè dalla loro storicità. ${ }^{10}$

Anche gli autori cristiani, teologi e storici da un lato, poeti dall'altro, favorivano una lettura e un'interpretazione dei testi non ancorata alla loro storicità: tipologia, allegoria e allegoresi in letteratura, teologia e storiografia vanno proprio in una direzione fondamentalmente anti-storicistica, in quanto esse invitano a interpretare il testo inserendo una frattura programmatica tra il momento storico originario della sua produzione e il suo intrinseco valore simbolico, figurale o allegorico. Ma questi metodi di lettura, per quanto ben noti agli studiosi, paradossalmente non vengono presi seriamente in considerazione, in quanto le interpretazioni che ne derivano sono considerate epistemologicamente instabili e non comprovabili dalla prospettiva offerta dal metodo fondamentalmente storicistico applicato dalla filologia classica, orientato cioè alla descrizione del testo e del suo contesto più che all'intepretazione. Eppure su di esse si basa gran parte della testualità tardoantica, che nella maggior parte dei casi viene invece letta unicamente attraverso la lente del passato classico, di cui l'applicazione del metodo intertestuale è senz'altro la marca più significativa, ma anche la più ideologica, proprio perché instaura surrettiziamente tramite la supposta continuità storica valori formali ed estetici che tendono a marginalizzare proprio gli aspetti di novità. Più in generale, anche se qualsiasi opera d'arte o testo è frutto ed espressione di un preciso momento storico, per sua natura recherà sempre con sé il riferimento ad un'origine ancenstrale a-storica, fuori dal tempo ('arcaica', secondo Agamben). E qualsiasi riferimento alle circostanze della propria creazione rischiano "di fare collassare l'opera nella propria storicità" ${ }^{11}$

Un altro aspetto dell'anacronicità che richiede attenzione è la riproducibilità, integrale o parziale, di un'opera (o testo) proveniente da un'altra epoca. Come affermano gli autori:

\footnotetext{
Nei secoli XV e XVI vennero concettualizzati diversi modelli di temporalità dell'immagine .... Un modello di particolare successo propose la perfetta intercambiabilità di un'opera con un'altra. Seguendo questo modello, un'opera non ripeteva semplicemente l'opera precedente in quanto la ripetizione avrebbe comportato una differenza strutturale. Piuttosto quella data opera semplicemente è l'opera precedente, così che la precedente non è più precedente ma presente. ${ }^{12}$
}

Nel Rinascimento ciò che distingue la produzione artistica sarebbe una riflessione diffusa e continua proprio intorno all'instabilità temporale dell'opera. Nagel e Wood affermano: "L'opera anacronizza, dal greco aná (di nuovo) e chronizein (essere tardo). Anacronizzare (to anachronize) equivale a 'essere nuovamente tardo', 'attardarsi'

10 Feeney (2007) 12-16

11 Nagel / Wood (2010) 9.

12 Ibid. 11. 
(to linger). L'opera è tarda innanzitutto perché segue una realtà che rappresenta, e poi anche quando quella rappresentazione (re-presentation) viene ripetuta in una situazione successiva. Quando l'opera è tarda, quando ripete, quando esita, quando ricorda, ma anche quando proietta un futuro o un ideale, è anacronica" ${ }^{13}$ Il termine viene introdotto come alternativa a 'anacronistico', che è judgemental, contiene cioè un giudizio morale sull'opera d'arte. Tale giudizio proviene dalla visione storicistica secondo la quale ogni evento, ogni opera e ogni testo ha un'unica collocazione possibile e oggettiva all'interno di una concezione del tempo fondamentalmente lineare. A differenza dell'anacronismo, l'opera anacronica si muove liberamente nel tempo e la sua natura non dipende da una concezione stabile della forma come ancorata alla storia. Descrivere un'opera come anacronistica significa concepire quell'opera non come arte ma piuttosto come testimonianza di un preciso momento storico o come un'inalienabile traccia della storia: il termine anacronismo cerca di dirci cosa un'opera è realmente (what the artwork really is). Descrivere invece un'opera come 'anacronica' equivale a dire ciò che l'opera d'arte fa in quanto arte (to say what the artwork does qua art). ${ }^{14}$ Secondo la teoria anacronica quindi l'attenzione normalmente rivolta al momento originario della produzione di un'opera d'arte (o di un testo), cioè a dire alla sua storicità, viene implicitamente messa in crisi dall'aspirazione, che ogni opera o testo reca, a essere universale e ad attraversare il tempo attraverso una catena di sostituzioni infinite. "Il modello sostitutivo - affermano Nagel e Wood - non era una concezione primitiva o dettata dalla superstizione, ma un modello di produzione in grado di cogliere in modo assai più netto del modello autoriale (cioè storico) la temporalità strana e molteplice di un'opera d'arte. Sostituzione e performance non devono essere viste come fasi successive l'una all'altra, ma piuttosto come due modelli di creatività in perenne competizione l'uno con l'altro. L'uno definisce e risponde all'altro. La performance autoriale asserisce la differenza della puntualità dell'evento contro ripetizione e continuità; la sostituibilità propone un'identità pur attraverso le differenze". ${ }^{15}$

Ovviamente la teoria dell'anacronicità non si esaurisce in queste poche considerazioni tratte dall'introduzione all'importante volume. Questi riferimenti, pur essendo stati qui presentati in maniera un po' rapsodica, offrono tutta una serie di assonanze, forse inaspettate, con un certo modo di accostarsi alla letteratura e alla cultura tardoantica. Soprattutto, essi servono a impostare la questione della loro specificità da una prospettiva originale e ben più ampia di quanto abbia fatto l'approccio tradizionale, orientato soprattutto allo studio dei fenomeni intertestuali. Il tardo antico, se indagato seguendo il percorso tracciato dall'anacronicità, mostra un'originalità sorprendente, difficile a cogliersi proprio per l'intrinseco rapporto che esso intrattiene con la tradizione letteraria provenienti dalle epoche precedenti.

13 Ibid. 13.

14 Ibid. 14.

15 Ibid. 16 


\section{Il Anacronicità e sostituibilità dell'evento in Ammiano Marcellino e nei Panegyrici Latini}

I due testi da me selezionati costituiscono solo un primo campione di prova, per così dire, su cui saggiare la teoria dell'anacronicità. La scelta è dettata dalla strana simbiosi, tutta tardoantica, tra due generi letterari apparantemente diversi e persino opposti: storiografia e panegirico. L'una è detta rappresentare la realtà storica sulla base della veridicità e dell'imparzalità, mentre l'altro è tacciato di praticare la menzogna e di distorcere quindi la realtà storica allo scopo di adulare il potente. Eppure, a ben guardare, il ruolo del passato assume in entrambi un ruolo fondamentale: attorno ad esso si articola infatti l'argomentazione e il discorso del genere stesso, non solo nell'opera storiografica, com'è ovvio (ma non così ovvio, dopo tutto), ma anche nel panegirico. Non sarà necessario in questa sede soffermarsi a lungo sui pochi passaggi selezionati, in quanto si tratterà principalmente di indicare una prospettiva derivante dalle considerazioni sopra esposte che possa offrire un approccio nuovo e, a mio parere, più consono alla particolare testualità tardoantica.

Le Res gestae di Ammiano Marcellino furono concepite come continuazione delle Historiae di Tacito, che vanno sino a Nerva (96 d.C.). Della sua opera tuttavia ci sono giunti solo i libri da 14 a 31, che narrano la storia dal regno di Costanzo II alla battaglia di Adrianopoli nel 378. Cioè a dire, i primi 13 libri contenevano circa 250 anni di storia, mentre gli ultimi quattordici appena 25 anni, gli anni cioè in cui Ammiano stesso ha vissuto. Già solo per questo motivo strutturale le Res Gestae possono considersi una sorta di opera autobiografica. La sensazione di leggere un'autobiografia è in realtà dovuta soprattutto al fatto che lo storico si esprime, più di qualsiasi altro suo predecessore greco o romano, nella prima persona. E proprio il fatto che Ammiano abbia detto moltissimo su se stesso ha suscitato molte perplessità: tipico il giudizio di Erich Auerbach, che descrive lo stile di Ammiano come barocco, dai tratti paradossali e foschi "In Ammiano - egli scrive - arretrano il sentimento umano e la ragione e rimangono solo il magico e il grottesco, e fa meraviglia che un alto ufficiale, attivo e serio, che ha passato gran parte della sua vita in dure campagne di guerra, abbia volto il suo talento a materia simile". ${ }^{16} \mathrm{La}$ lettura di Auerbach è stata messa radicalmente in discussione negli ultimi decenni, ma il suo nucleo continua ad affascinare e, in parte, anche a convincere proprio per la specificità che egli accorda al testo di Ammiano come prova dei cambiamenti estetici nella tarda antichità. Ammiano, ben più degli altri storici antichi, greci e romani, ha basato la sua opera storiografica sull'autopsia, il metodo storiografico per eccellenza la cui elaborazione risale alle origini del pensiero storico occidentale. Eppure, proprio questo elemento così tradizionale acquista in Ammiano uno spessore ben diverso, che ha confuso e tuttora confonde gli interpreti. Non è qui il caso di

16 Auerbach (1956) 61-62. Sullo stile barocco di Ammiano secondo Auerbach cf. Stenger (2014). 
fare un riassunto dei pareri critici, ma è interessante notare come su Ammiano siano state espresse opinioni molto contrastanti: chi lo ha elogiato come storico sensibile ed esperto, teoricamente fondato (John Matthews), chi lo ha definito, con un po' di ironia, un "novelist”, un romanziere (Timothy Barnes), che non è veramente in grado di fornire una descrizione storica accurata, guidato semmai da forti "passioni e pregiudizi”, ben lontano dagli standard di obiettività che si devono adottare da parte di uno storico di professione. E anche Arnaldo Momigliano su Ammiano scrisse che lo storico mostrava "una sorta di indecente prontezza a parlare di se stesso". ${ }^{17}$

Un nuovo modo di accostarsi a questo testo è stato proposto da Gavin Kelly nel suo libro Ammianus Marcellinus. The allusive historian (2008). Come è possibile comprendere già dal titolo, Kelly vuol leggere le Res gestae come un'opera animata da una profonda continuità con la tradizione letteraria romana. Infatti, il testo di Ammiano, secondo Kelly, dal punto di vista della tecnica allusiva presenta le stesse caratteristiche delle grandi opere poetiche e in prosa della tradizione letteraria greca e romana. Inoltre, il critico si propone di superare il biografismo che caratterizza molti dei contributi precedenti. ${ }^{18}$ In effetti, la tendenza principale è stata di discutere le Storie ammianee con l'intenzione di verificare se e sino a che punto l'autore sia stato veramente presente agli eventi descritti, se le sue fosche e spiazzanti descrizioni, tanto apprezzate da Auerbach, siano davvero il risultato di un'osservazione diretta e di una reale partecipazione agli eventi. Kelly si contrappone a questa tendenza spostando l'attenzione sull'analisi intertestuale al fine di dimostrare fondamentalmente che le Res Gestae, anche là dove riportano le esperienze vissute in prima persona dall'autore, sono un testo letterario, che andrà letto e valutato più sulla base della sua testualità che non della veridicità o fondatezza delle descrizioni in esso riportate. Infine, egli è dell'opinione che proprio l'analisi intertestuale dell'opera non solo non offuschi la fruizione storico-politica dell'opera ma la renda ancora più interessante e complessa esattamente da quel punto vista. ${ }^{19}$

Nelle prossime pagine proverò ad aprire la via a un altro tipo di interpretazione, che non mostra corrispondenza alcuna con la biografia e le intenzioni dell'autore ${ }^{20} \mathrm{e}$ che al contempo è orientata a cogliere la specificità dell'estetica tardoantica concentrandosi su un modello di temporalità, che sulle orme di quanto esposto nella prima parte di questo contributo, definisco come 'anacronica'. Qui discuto in particolare un passo tratto dall'opera di Ammiano, che ha già ricevuto grande attenzione da parte di vari studiosi, e in particolare proprio da Kelly nel lavoro appena menzionato. Nel libro 18 Ammiano racconta le vicende riguardanti la spedizione contro i

17 Matthews (1989), Barnes (1998), Momigliano (1980) 144.

18 Biografismo di cui non è, tuttavia, privo nemmeno Kelly stesso, si veda per es. Kelly (2007).

19 Kelly (2008) 30.

20 Concordo con Kelly nel respingere il biografismo dominante nelle letture di Ammiano del secolo scorso. Mi sembra tuttavia che anche Kelly ricada nella falla del biografismo e dell'intenzionalità allorquando insiste sui risvolti politici della tecnica allusiva adottata dallo storico tardoantico. 
Persiani comandata da Ursicino e di cui egli stesso fa parte. Ad un certo punto Ammiano riferisce su un'azione di spionaggio che lo vede protagonista.

Erat eo tempore Satrapa Corduenae, quae obtemperabat potestati Persarum, Iovinianus nomine appellatus in solo Romano adulescens, nobiscum occulte sentiens ea gratia, quod obsidatus sorte in Syriis detentus et dulcedine liberalium studiorum inlectus remeare ad nostra ardenti desiderio gestiebat. 21. ad hunc missus ego cum centurione quodam fidissimo exploratius noscendi gratia, quae gerebantur, per avios montes angustiasque praecipites veni. visusque et agnitus comiterque susceptus causam praesentiae meae uni illi confessus adiuncto taciturno aliquo locorum perito mittor ad praecelsas rupes exinde longe distantes, unde nisi oculorum deficeret acies, ad quinquagesimum usque lapidem quodvis etiam minutissimum apparebat. 22. ibi morati integrum biduum, cum sol tertius adfulsisset, cernebamus terrarum omnes ambitus subiectos, quos horizontas appellamus, agminibus oppletos innumeris et antegressum regem vestis claritudine rutilantem. quem iuxta laevus incedebat Grumbates Chionitarum rex novus aetate quidem media rugosisque membris sed mente quadam grandifica multisque victoriarum insignibus nobilis; dextra rex Albanorum pari loco atque honore sublimis; post duces varii auctoritate et potestatibus eminentes, quos ordinum omnium multitudo sequebatur ex vicinarum gentium roboribus lecta, ad tolerandam rerum asperitatem diuturnis casibus erudita. 23. Quo usque nobis Doriscum Thraciae oppidum et agminatim intra consaepta exercitus recensitos Graecia fabulosa narrabis? cum nos cauti vel, ut verius dixerim, timidi nihil exaggeremus praeter ea quae fidei testimonia neque incerta monstrarunt. ${ }^{21}$

A questo testo Kelly ha dedicato un'analisi molto accurata e convincente, mirata soprattutto a ricostruire il tessuto di rimandi intertestuali rielaborati dallo storico. ${ }^{22} \mathrm{E}$ in effetti, il passo ben rappresenta il linguaggio testuale di Ammiano. Contiene una descrizione di un evento strano, che il "lonely historian" (secondo la celebre defi-

21 18.6.20 - 23: "era in quel tempo satrapo della Corduene, territorio posto sotto il dominio persiano, un giovane che dai Romani veniva chiamato Gioviniano. Questi nutriva segrete simpatie nei nostri confronti perché, trattenuto come ostaggio in Siria ed affascinato dalla bellezza degli studi liberali, desiderava ardentemente ritornare fra noi. Presso di lui fui inviato assieme ad un centurione fidatissimo, per conoscere a fondo ciò che i nemici facevano, e vi giunsi attraverso montagne impervie e strette gole. Vistomi e riconosciutomi, mi accolse gentilmente ed a lui solo confidai la ragione della mia visita. Accompagnato da un silenzioso conoscitore di quella zona, fui mandato su alcune rupi altissime, molto distanti da quel luogo, donde si poteva vedere, a meno che la vista non fosse mancata, ogni più piccolo particolare per un raggio di cinquanta miglia. Ci trattenemmo in questa località per due giorni interi ed il terzo giorno, al sorgere del sole, vedemmo sotto di noi tutta la regione nella sua ampiezza, sino a quelli che noi chiamiamo ópí̧ovtعs [sic], piena di innumerevoli schiere in testa alle quali procedeva il re rilucente per lo splendore della veste. Alla sua sinistra s'avanzava il re dei Chioniti, Grumbate, uomo di forza media e dalle membra rugose, ma dotato di viva intelligenza e famoso per numerose e celebri vittorie. Alla sua destra era invece il re degli Albani, a lui pari di grado e fatto oggetto di onore altissimo. Seguivano numerosi comandanti, insigni per autorità e potere, a cui tenevano dietro militari d'ogni grado in gran numero, scelti fra il fiore delle popolazioni vicine ed esercitati da una serie di eventi ad affrontare l'asprezza della lotta. Fin quando, o Grecia che ami le favole, ci narrerai di Dorisco, città della Tracia, e degli eserciti contati a schiere entro palizzate? Noi invece, cauti o, per dire il vero, pieni di timore, non esageriamo con affermazioni che vadano oltre le prove fondate su testimonianze degne di fede e sicure" (trad. Selem).

22 Kelly (2008) 30. 
nizione di Momigliano) narra in prima persona e con una particolare attenzione al proprio punto di vista. Egli vi fa un implicito riferimento alle sue origini greche (e, per converso, a una certa estraneità alla lingua latina che pure padroneggia) e, elemento decisivo per Kelly, vi riprende come exemplum un episodio tratto da Erodoto, marcando così in modo evidente l'allusività della sua tecnica storiografica. La complessità del passo emerge soprattutto in alcuni dettagli, su cui è opportuno soffermarsi brevemente. Da un lato si legge un'insistenza sulla testimonianza diretta e l'esperienza autoptica di cui Ammiano va fiero, dall'altro si invoca, anche se in modo contraddittorio, l'autorità della storiografia stessa ai suoi venerabili esordi. L'esperienza narrata sconfina nel meraviglioso e, paradossalmente, nell'incredibile. Ammiano con una tecnica raffinata prepara letteralmente il terreno alla 'visione' che avrà luogo poco dopo: egli prima "attraverso montagne impervie e strette gole" raggiunge il suo informante (di cui svela la passione per le artes liberales), dopo viene mandato ad praecelsas rupes, postazione ideale per osservare il territorio circostante. L'accompagnatore a cui viene affidato è taciturnus. Da un punto di vista narrativo il silenzio, eliminando il coinvolgimento di uno dei sensi, è funzionale a esaltare la vista; come se la parola si dileguasse per lasciare spazio alla vista. L'autopsia diviene l'unica protagonista, sufficiente a se stessa. Ma poi la frase: unde nisi oculorum deficeret acies, ad quinquagesimum usque lapidem quodvis etiam minutissimum apparebat. Come fa presente Kelly, traduce bene Guy Sabbah: apparebat "sarebbe potuto apparire". ${ }^{23}$ Quindi: "se la vista umana non fosse troppo debole di natura, si sarebbe potuto vedere ogni più piccolo particolare per un raggio di cinquanta miglia”. E subito dopo, Ammiano racconta di essersi trattenuto due interi giorni, e al sorgere del terzo (sol tertius affulsisset), egli poteva scorgere tutto il territorio nella sua ampiezza sino a quello che egli, da greco, chiama "orizzonte" (quos horizontas appellamus), sul quale si distendevano le schiere dell'esercito persiano in tutto lo splendore delle armi e delle vesti. Dopo avere descritto l'ordine in cui le schiere erano disposte, Ammiano inserisce un commento personale - e metatestuale -, che rompe in modo abrupto la narrazione storica, trasferendo quanto fin qui narrato in una dimensione che definirei sovrastorica: Quo usque nobis ... Graecia fabulosa narrabis? "Fin quando, oh Grecia che ami le favole, ci racconterai della trace Dorisco e degli eserciti contati a schiere entro le palizzate?” La vista come categoria storiografica (autopsia) rappresenta il tema profondo del passo in questione (si notino i numerosi termini che vi fanno riferimento). Alla pretesa autoptica della voce del narratore viene contrapposta la narrazione storiografica tradizionale. Infatti, dietro la fabulosa Graecia si nasconde un passo di Erodoto (7.60), che, non essendo qui nemmeno nominato, viene apparentemente destabilizzato nei suoi fondamenti. Una strana tensione percorre questo testo, difficilmente comprensibile sino in fondo. È come se le coordinate spaziali e temporali si fondessero. Si notino a tal proposito i vari termini (in grassetto nel testo), che sottolineano la dimensione

23 Ibid. 82 n. 149. 
autoptica attraverso riferimenti spaziali e temporali. In particolare le frasi centrali del passo descrivono implicitamente l'instabilità della vista stessa: nisi oculorum deficeret acies ... cum sol tertius adfulsisset ... cernebamus ... quos horizontas appellamus ... claritudine rutilantem. Sembra quasi che la vista di Ammiano, e con lui del suo lettore, venisse come sfidata dalla distanza, dallo splendere del sole, dal limite oggettivo dato dall'orizzonte, dalle vesti scintillanti del re. Un altro elemento spiazzante è dato dal commento finale al passo: "Noi invece, cauti o, per meglio dire, timorosi (timidi), non esageriamo con affermazioni che vadano oltre le prove fondate $\mathrm{su}$ testimonianze (fidei testimonia) degne di fede e sicure (neque indubia, neque incerta)". Che può voler dire questa frase? Kelly indica che, se da un lato Ammiano vuole proclamare la veridicità della propria narrazione che si basa sull'autopsia, dall'altro egli non vuole negare l'importanza di fonti di autorità alternative, cioè il ricorso ad altri testimoni, limitando il valore dell'autopsia stessa ma accrescendo il peso della voce delle fonti, fissate come exempla. L'exemplum addotto in questo caso ha una funzione piuttosto singolare: conferma sì la realtà attuale come descritta da Ammiano, ma come per contrasto. Il passo erodoteo in questione, marchiato con l'aggettivo fabulosum, è sottoposto ad un processo di verifica attraverso l'esperienza attuale, da cui viene messo in crisi e confermato a un tempo. È come se Ammiano dicesse: leggete Erodoto, troverete che ciò che egli racconta sia semplicemente incredibile. Eppure io stesso ho visto qualcosa di molto simile a quell'evento in questa precisa circostanza e ve lo racconto con una dovizia di particolari maggiore che in Erodoto stesso. Paradossalmente il lettore è come invitato a credere proprio a quanto l'autore ha bollato come fabulosum, incredibile. La conferma si trova in un passo successivo: è come se Ammiano correggesse quanto lui stesso aveva affermato precedentemente. ${ }^{24}$ Ancora una volta si riferisce alla percezione di Erodoto, diffusa nell'antichità, come un narratore di eventi favolosi piuttosto che di storia, e afferma che esattamente ciò che nelle Storie erodotee si reputava essere incredibile, cioè l'immensità delle truppe persiane, si è poi rivelato possibile durante l'invasione gotica del 376 quando si vide un'enorme massa di soldati.

Resipiscant tandem memoriae veteres, Medicas acies ductantes ad Graeciam: quae dum Hellespontiacos pontes et discidio quodam fabrili mare sub imo Athonis pede quaesitum exponunt et turmatim apud Doriscum exercitus recensetos, concordante omni posteritate ut fabulosae sunt lectae. nam postquam innumerae gentium multitudines per provincias circumfusae, pandentesque se in spatia ampla camporum, regiones omnes et cuncta opplevere montium iuga, fides quoque vetustatis recenti documento firmata est. ${ }^{25}$

24 Kelly (2008) 86.

25 31.4.7-8: "Riprendano animo infine le antiche storie che narrano l'arrivo delle schiere dei Medi, narrazioni che, per concorde giudizio di tutti i posteri, furono ritenute favolose là dove trattano dei ponti sull'Ellesponto, del mare che, con un taglio compiuto da operai, fu cercato ai piedi dell'Athos, e degli eserciti contati a squadre nei pressi di Dorisco. Infatti, dopo che innumerevoli moltitudini, sparsesi tutt'attorno per le province e diffusesi per le ampie distese dei campi, riempirono tutte le 
Il testo di Ammiano è anacronico, in quanto sostituisce la propria visione a quella narrata da Erodoto secoli prima. Riprendendo le considerazioni esposte nella prima parte di questo saggio, l'evento che Ammiano racconta nei suoi termini non è una semplice ripetizione dell'evento riportato da Erodoto, e, pace Kelly, non è soltanto un'allusione, ma è quell'evento stesso, vi si riallaccia senza soluzione di continuità, come sostituendolo nella memoria storica. Come affermavano sopra Nagel e Wood, un'opera è tarda quando propone una rappresentazione (re-presentation) di qualcosa che viene ripetuto in una situazione successiva. Una vertiginosa continuità è qui stabilità tra un passato remoto e un fatto contemporaneo, il primo semplicemente rivive nell'altro, sostituendolo in una profonda unità concettuale. Analogamente alla reazione che ebbe Beauvoir di fronte al Palazzo Imperiale cinese, che non esibisce la sua storicità e che viene perciò descritto dalla scrittrice come un'“imitazione di se stesso", la perplessità dei lettori odierni di Ammiano va presa seriamente in considerazione in quanto tale, e non respinta come un errore da correggere tramite l'accurata ricostruzione dei modelli testuali a cui Ammiano allude. L'allusione non è un atto sempre uguale a se stesso, cambia la sua funzione e, soprattutto, per quanto precisa sia la sua descrizione, essa va interpretata. Si tratta con tutta evidenza di un atto di presentizzazione di un passato arcaico, che dà accesso alla conoscenza del presente in modo intuitivo ma non per questo meno efficace. L'evento di cui lo storico tardoantico è testimone diventa al contempo il suo arcaico e tipologico predecessore, come narrato in Erodoto. Il testo di Ammiano diviene così anche anacronico, in grado cioè di eludere la propria storicità riallacciandosi ad un passato remoto che torna a rivivere, ma non come ricostruzione storica né come semplice analogia, piuttosto come il manifestarsi di una conoscenza lampeggiante che non conosce i confini stabiliti dalla temporalità storica. Nei termini di Benjamin e Agamben, siamo di fronte al manifestarsi di un Ursprung, di un momento 'arcaico' in quanto ripropone l'arché (origine) di se stesso.

Come è stato opportunamente messo in luce da Kelly e da altri studiosi, ${ }^{26}$ l'opera di Ammiano intreccia continuamente la narrazione dei fatti, di cui l'autore è testimone, con il ricordo di fatti storici come descritti da altri storici, per lo più Sallustio e Tacito, ma anche in tanti altri testi appartenenti ai più disparati generi letterari, dalla satira alla poesia epica alla tragedia. Come sottolinea Kelly, le Res Gestae presentano un vero e proprio impianto enciclopedico, la sua intertestualità è "vorace e promiscua". ${ }^{27}$ Il testo appare come un immane centone, in cui si uniscono mille voci del passato, sia greco che romano. In un recente articolo Martin Hose prende spunto dal discorso intorno alla crisi della rappresentazione storiografica, come esso si è andato delineando nel dibattito teorico sin dall'opera di Hayden White per concentrarsi sull'intertestualità ammianea, in particolare sull'impiego di exempla e proverbi, a cui

regioni e tutti i gioghi delle montagne, anche la veridicità degli storici antichi è stata dimostrata con una prova recente" (trad. Selem).

26 Si veda in particolare la monografia di Wittchow (2001).

27 Kelly (2008) 214. 
Ammiano ricorre spessissimo proprio per spiegare ciò che non sembra spiegabile e che gli eventi del suo proprio tempo non gli lascerebbero comprendere. ${ }^{28}$ Se le opere storiografiche precedenti di regola interpretavano il passato alla luce del presente, Ammiano ribalta la prospettiva, indicando che è necessario leggere il presente alla luce del passato, come appunto testimonia l'amplissima utilizzazione di exempla e proverbi. ${ }^{29}$ I fittissimi riferimenti intertestuali, di cui le Res gestae risuonano, non rappresentano per Hose una pura dimensione estetica (così come egli pensa sia presso Kelly), ma offrono l'unica possibilità per comprendere la storia, in particolare i suoi aspetti incommensurabili e non direttamente comprensibili. Ammiano diviene così un rappresentante della crisi del discorso storiografico ante litteram, in quanto "conduce in una costellazione, nella quale ciò che nel presente può essere discusso solo su un livello metadiscorsivo - la problematizzazione cioè del rapporto tra ciò che viene rappresentato e la rappresentazione stessa - può e deve divenire oggetto di negoziazione nel discorso storiografico nella sua concretezza". ${ }^{30}$ Hose, reagendo all'analisi intertestuale condotta da Kelly, che lui reputa un po' troppo formale, richiama l'attenzione sul punto nodale della storiografia stessa, cioè il rapporto tra la realtà storica e i mezzi per rappresentarla. Egli vede così nel testo di Ammiano un importante trait d'union tra una specifica estetica tardoantica e la necessità di un rinnovamento della storiografia, entrambe da lui collocate sotto l'egida della tendenza ermeneutica, che in età tardoantica predomina sulla mimesi (come già era stato messo in rilievo da Herzog, che aveva parlato della tarda antichità come di un'aetas hermeneutica). ${ }^{31}$

Sin qui non posso che essere d'accordo con Hose, soprattutto perché questi, a differenza di Kelly, nella sua analisi svela e mette in luce un aspetto importante dell'estetica tardoantica attorno a cui costruisce la propria argomentazione. Tuttavia, quanto da lui affermato non riguarda soltanto il discorso storiografico ma è ravvisabile in un numero di testi ben più ampio, non solo in prosa ma anche in poesia.

Per dimostrare come gli aspetti messi in luce da Kelly e da Hose siano diffusi anche in altri tipi di testo, scelgo di soffermarmi brevemente su un genere tipicamente tardoantico, che, rappresenta, per così dire, la spina al fianco della storiografia, in quanto esso sfida e contrasta il discorso dello storico proprio nei suoi punti più problematici: il panegirico, ${ }^{32}$ in particolare come esso si delinea all'interno dei così detti Panegyrici Latini. Si tratta di un corpus contenente dodici discorsi scritti, e in parte recitati, in onore di diversi imperatori, dalla gratiarum actio resa da Plinio il Giovane a Traiano al panegirico composto da Latino Pacato Drepanio per Teodosio il

28 Hose (2015) 84: “Um Nichtverstehbares verstehbar zu machen”.

29 Ibid. 87.

30 Ibid. 88.

31 Herzog (1989).

32 Per una discussione generale intorno alla pervasività della dimensione panegiristica in età tardoantica si veda Formisano (2012). 
Grande nel $389 .^{33}$ In questa sede non è possibile entrare nel dettaglio né sulla data dei singoli discorsi, né su quella di composizione della collezione, che va presumibilmente collocata alla fine del quarto secolo, nello stesso periodo quindi della pubblicazione dell'opera di Ammiano. Mi limito a indicare una caratteristica, la cui percezione si rivela a parer mio fondamentale a chi si accosta al corpus: se ad ogni singolo discorso è legittimo attribuire un significato storico preciso, emanante dalle circostanze in cui ognuno di essi è stato composto, la raccolta in quanto tale elude, direi programmaticamente, questa possibilità. Non solo. Se il significato di ogni singolo discorso è la lode dell'imperatore di volta in volta celebrato, il corpus nella sua polifonia, per tutta una serie di motivi, destabilizza e sovverte la figura dell'elogiato. Cioè a dire: il significato emanante dal macrotesto è in contraddizione con quello dei singoli discorsi. ${ }^{34}$ I Panegyrici Latini, se letti dalla prospettiva macrotestuale, si rivelano una fonte preziosissima per indagare la testualità del periodo tardoantico, circostanza meno ravvisabile allorquando si considerano i testi nella loro individualità, isolatamente gli uni dagli altri e come essi sono stati composti e recitati in prima istanza, tendenza, questa, che tutt'ora caratterizza gran parte dei contributi critici. In particolare, vorrei fare qui riferimento a un aspetto già sopra accennato: la collezione presenta il panegirico come un attivo concorrente della storiografia. Infatti esso arrogandosi di contenere la vera storia, che è sempre quella presente, sottrae credibilità ai testi storiografici, di cui destabilizza sistematicamente le categorie epistemiche. E ciò avviene, un po' paradossalmente, in quanto i panegiristi ricorrono a un tipo di argomentazione del tutto simile a quella che abbiamo visto in Ammiano nel passo appena ricordato e in alcuni altri. Si veda per esempio un passo tratto dal panegirico di Nazario a Costantino:

Estote, o gravissimi auctores, de scriptorum religione securi: credimus facta qui maiora nunc sensimus. Magnitudo principis nostri gestis veterum fidem conciliat, sed miraculum detrahit. ${ }^{35}$

Qui gli "autori venerandi" sono gli storici, a cui il panegirista dichiara di credere proprio perché egli stesso è testimone di fatti ancora più sorprendenti. Come in un epigramma, un'arguta pointe corona il ragionamento: le imprese di Costantino da un lato confermano che quanto raccontato dagli storici è realmente avvenuto, ma al contempo per la loro stessa magnificenza sottragono loro ogni sacralità e prodigiosità (miraculum).

33 Sui Panegyrici Latini si veda McCormack (1976), Rees (2002), Formisano (2008), Rees (2012), Gibson / Rees (2013).

34 Sul concetto di macrotesto e della sua applicazione a testi latini si veda Formisano (2016a) e Id. (2016b).

35 Paneg. 4.15.6: "Ma state tranquilli, o scrittori autorevoli, rispettiamo i vostri scritti, non abbiamo difficoltà a credere ai fatti da voi raccontati, noi che ne abbiamo visti di più grandi. Ma la grandezza del nostro principe, se da una parte induce ad avere fiducia nelle imprese degli antichi, dall'altra, però, toglie loro ogni prodigiosità" (trad. Lassandro). 
Nella gratiarum actio di Claudio Mamertino all'imperatore Giuliano si trova un riferimento alla letteratura greca dal piglio analogo a quello che abbiamo già osservato in Ammiano, anche se qui non è un testo storico a essere preso di mira, ma un mito come probabilmente narrato nelle Argonautiche di Apollonio Rodio.

O facundia potens Graecia! Omnium tuorum principum gesta in maius extollere sola potuisti, sola factorum glorias ad verborum copiam tetendisti. Tu navem unam propter aurati velleris furtum et virginis raptum in caelum usque sublatam sideribus consecrasti .... Quid tu, si ad scribendas celebrendasque res principis nostri animum adieceris, de Iuliani lembis liburnisque factura es? ... Qua dignitate describes classem per maximi fluminis tractum remis ventisque volitantem, tum principem nostrum alta puppi sublimen ...? $?^{36}$

La Grecia stessa viene apostrofata in quanto "potente nell'arte del dire" e al contempo sfidata: sarebbe in grado lei, che ha saputo narrare le vicende degli Argonauti rendendoli immortali, di descrivere le imprese navali di Giuliano? In questo caso il panegirista tratta il presente come un mito e, al contempo, sottrae sacralità al mito stesso, che viene come ridicolizzato al confronto con le clamorose gesta dell'attuale imperatore.

Un altro Mamertino in un precedente discorso, reso in occasione del genetliaco di Diocleziano e Massimiano, si scaglia contro le fabulae degli ignoranti, insistendo che quanto lui afferma è la sola verità: Sed removeamus istinc fabulas imperitorum, verum loquamur: vestra vobis pietas, sacratissime imperator, volucres dedit cursus. (Paneg. 11.8.4-5: "Ma via di qui le favole degli ignoranti, e diciamo come sono andate veramente le cose: la pietà che vi portate l'un l'altro ha messo le ali, o sacratissimo imperatore, alla vostra corsa" [trad. Micunco]). Nell'anonimo discorso reso a Massimiano in occasione dell'anniversario della fondazione di Roma si trova un analogo pensiero: Omittam cetera, et potissimum illud adripiam quod multis fortasse mirum videbitur et tamen re ipsa verissimum est (Paneg. 10.3.1: "tralascerò il resto e coglierò la cosa più importante, che a molti forse sembrerà incredibile, ma che corrisponde alla realtà più vera" [trad. Micunco]). Per quanto ciò che il panegirista narra possa sembrare miracoloso, e quindi incredibile, questa è la pura verità, a cui ci costringe a credere, nonostante egli stesso implicitamente la destabilizzi.

Anche Pacato Drepanio, a cui sono in molti ad attribuire la paternità della collezione, ${ }^{37}$ si esprime in un linguaggio che sembra dialogare direttamente con il

36 Paneg. 3.8.1 ss.: “O Grecia, signora dell’eloquenza! Tu sola hai potuto grandemente esaltare le imprese di tutti i tuoi capi, tu sola sei riuscita a sviluppare le potenzialità della parola per glorificare degnamente le imprese. Tu hai consacrato, innalzandola fino agli astri del cielo, una sola nave per il furto del vello d'oro e il rapimento di una fanciulla. ... Cosa faresti, se dovessi porre mente a scrivere e celebrare le imprese del nostro principe? come penseresti di trattare delle imbarcazioni e delle liburne di Giuliano? ... Con quale dignità descriveresti la flotta che, spinta dai remi e dai venti, vola lungo il corso del più grande dei fiumi; e il nostro principe, ritto sull'alto della poppa ...?” (trad. Micunco).

37 Da Pichon (1906) a Rees (2013). 
testo di Ammiano. Il suo panegirico, composto nel 389 in onore di Teodosio (e quindi probabilmente contemporaneo alla pubblicazione dell'opera di Ammiano), chiude cronologicamente la raccolta, anche se vi è stato inserito come secondo, subito dopo quello di Plinio il Giovane, a mio parere per esaltarne il contrasto più che le affinità. Si veda in particolare un passo, nel quale l'autore tematizza la credibilità del proprio testo rispetto alle narrazioni provenienti dalla tradizione.

Ego vero, si caeleste studium pro dignitate causarum aestimandum sit, iure contenderim equites tuos Pegasis, talaribus pedites vectos ac suspensos fuisse. neque enim quia se divina mortalibus dedignantur fateri, idcirco quae visa non fuerint dubitabimus facta, cum facta videamus quae dubitaverimus esse facienda. ${ }^{38}$

Ad essere trattata è ancora una volta la sfera del mito. Con un'accortezza tipica del linguaggio dei panegiristi, anche Pacato evita il riferimento ad una religione precisa, sottolineando tuttavia che "le cose divine" (divina) non si svelano ai mortali, e tuttavia è necessario credere a quanto affermano le storie in quanto oggi si verificano fatti - le gesta dell'imperatore - ai quali risulta assai difficile prestare fede. Il concetto finale è espresso con un linguaggio particolarmente barocco e concettoso, così che esso, paradossalmente, finisce per confondere, sottraendo così credibilità a quanto appena affermato.

Come si vede nei pochi passi selezionati, alla storia viene accostato il mito. Non solo: la dimensione mitica in realtà viene come presentizzata e sostituita alla storia stessa, che a sua volta viene come fizionalizzata, resa cioè finzione letteraria. I pochi passagi tratti dai Panegyrici Latini non offrono che uno sguardo superificiale rispetto all'abbondanza delle rappresentazioni della storia e dell'antiquitas contenute nell'intera raccolta. In questa sede sia sufficiente sottolineare come il lettore assista a una duplice sostituzione: da un lato il presente sostituisce il passato, stravolgendone il valore esemplare normalmente attribuitogli, dall'altro innalza il mito a categoria storica, a cui si deve prestare fede. Da un punto di vista formale, non è il mito che viene storicizzato, ma è la storia ad essere rappresentata come mito, in modo analogo, quindi, a quanto osservato da Hose, secondo il quale in Ammiano il presente viene letto e interpretato attraverso la lente del passato. ${ }^{39}$ Un atto puramente anacronico, in quanto il testo si configura come risultante da una catena di sostituzioni, sia nel contenuto, cioè nei fatti descritti, sia nella forma, cioè nel processo di mitizzazione della realtà storica, che, paradossalmente, viene resa ancora più 'incredibile' del mito stesso.

38 Paneg. 2.39.5: "Ma io, se devo valutare i favori celesti in base alla dignità delle cause che li determinano, credo di poter a buon diritto sostenere che i tuoi cavalieri furono portati in volo da dei Pegasi e i tuoi fanti da calzari alati. Le realtà divine non si degnano di rivelarsi ai mortali; ma non per questo dubiteremo che siano veramente avvenuti fatti che non abbiamo potuto vedere, se vediamo avvenuti fatti che potremmo dubitare che dovessero accadere" (trad. Micunco).

39 Sul ruolo predominante del presente, che ha come conseguenza una sorta di azzeramento del tempo storico, si veda Herzog (1980). 
Il tardoantico è stato definito come un "zeitloses und geschichtsloses Zeitalter" un'epoca priva di storicità, già da Herzog, poi da Eigler nella monografia citata in apertura. ${ }^{40} \mathrm{Si}$ assiste infatti ad una sorta di vertiginosa sospensione temporale diffusa in molti testi, anche in quelli storiografici. Ho parlato di Ammiano, ma si pensi anche a Orosio. ${ }^{41}$ La ripetitività e sostituibilità del passato accompagnano molte delle silenziose trasformazioni della cultura nella tarda antichità. Si pensi alla sostituzione della lectio vetustatis attraverso la lectio divina, ossia la lettura della Bibbia. Come nota Eigler, "i mores romani trovano conferma attraverso gli exempla biblici. La Bildung romana tradizionale e quella cristiana sono rappresentate da una stessa figura in una coesistenza priva di conflitto". ${ }^{42}$

Per concludere, l'età tardoantica, se osservata dalla prospettiva anacronica, si delinea come un'epoca che, forse sorprendentemente, non costruisce la propria identità culturale sulla storicità. Il passato che viene evocato è privo di tempo, zeitlos, e la cifra culturale dell'epoca è data proprio dalla sostituibilità del passato attraverso il presente. In un precedente contributo avevo affermato che la tarda antichità è l'epoca innominabile della cultura occidentale giacché entrambi i termini, tardo e antico, come anche accennato qui in apertura, sono fuorvianti. ${ }^{43} \mathrm{~Eb}$ bene, l'anacronicità ristabilisce precisamente il valore dell'essere tardo (belatedness) così che la definizione tarda antichità acquista un nuovo significato, in quanto il tardo non è più una semplice categoria temporale, che si voleva neutra, ma diventa una nuova categoria estetica, storica e interpretativa.

\section{Bibliografia}

Agamben (2009): G. Agamben, “Che cos'è il contemporaneo?”, in: Id., Nudità, Roma, 19-32.

Auerbach (1956): E. Auerbach, Mimesis. Il realismo nella letteratura occidentale, Torino.

Barnes (1998): T. D. Barnes, Ammianus Marcellinus and the Representation of Historical Reality, Ithaca / London.

Bažil (2013): M. Bažil “'Textus gestorum, factorum, rei totius'. Geschichte als 'textus' bei Ammianus Marcellinus”, in: B. Marek, P. Honč et al. (eds.), Visuque et auditu iuxta venerabilis adrogantiam effugerat. FS. prof. Bohumila Mouchová, Praha, 31-42.

Benjamin (1999): W. Benjamin, Il dramma barocco tedesco, Torino.

Derrida (1998): J. Derrida, Demeure. Maurice Blanchot, Paris.

Didi-Huberman (2007): G. Didi-Huberman, Storia dell'arte e anacronismo delle immagini, Torino. Eigler (2003): U. Eigler, lectiones vetustatis. Römische Literatur und Geschichte in der lateinischen Literatur der Spätantike, München.

Feeney (2007): D. Feeney, Caesar's calendar. Ancient time and the beginnings of history, Berkeley / Los Angeles / New York.

40 Herzog (1980) e Eigler (2003), 25 s.

$41 \mathrm{Su}$ Orosio rimando a Van Nuffelen (2012) e Formisano (2013).

42 Eigler (2003) 27.

43 Formisano (2014). 
Formisano (2007): M. Formisano, "Towards an Aesthetic Paradigm of Late Antiquity", Antiquité Tardive 15, 277-284.

Formisano (2008): M. Formisano, "Speculum principis, speculum oratoris. Alcune considerazioni sui panegyrici latini come genere letterario", in: L. Castagna and C. Riboldi (eds.), Amicitiae templa serena. Studi in onore di Giuseppe Aricò, Milano, 581-599.

Formisano (2012): M. Formisano, "Late Antiquity, New Departures", in: R. Hexter and D. Townsend (eds.), The Oxford Handbook of Medieval Latin Literature, Oxford, 509-534.

Formisano (2013): M. Formisano, “Grand Finale. Orosius' Historiae adversus paganos or the subversion of history", in: H. Harich-Schwarzbauer and K. Pollmann (eds.), Rome 410, Berlin / New York, 153-176.

Formisano (2014): M. Formisano, "Reading Décadence: Reception and the Subaltern Late Antiquity", in: M. Formisano and T. Fuhrer (eds.), Décadence. "Decline and Fall" or "Other Antiquity"?, Heidelberg, 7-16.

Formisano (2016a): M. Formisano, “Reading Dismemberment: Dinocrates and the Macrotext”, Arethusa 49, 145-160.

Formisano (2016b): M. Formisano, "La fabula Aristaei, l'aneddoto di Dinocrate e il macrotesto", in: A. Setaioli (ed.), Apis Matina. Studi in onore di Carlo Santini, Trieste, 306-318.

Gibson / Rees (2013): B. Gibson and R. Rees (eds.), Pliny the Younger in Late Antiquity, Arethusa 46.2.

Herzog (1980): R. Herzog, "Orosius oder die Formulierung eines Fortschrittskonzepts aus der Erfahrung des Niedergangs”, in: R. Koselleck and P. Widmer (eds.), Niedergang, Stuttgart, $79-102$.

Herzog (1989): R. Herzog, "Einführung in die lateinische Literatur der Spätantike”, in: R. Herzog (ed.), Restauration und Erneuerung. Die lateinische Literatur von 284 bis 374 n. Chr. (Handbuch der lateinischen Literatur der Antike, Band 5), München, 1-44.

Hose (2015): M. Hose, "Intertextualität als hermeneutisches Instrument in der spätantiken Literatur. Das Beispiel Ammianus Marcellinus”, in: J. Stenger (ed.), Spätantike Konzeptionen von Literatur, Heidelberg, 75-90.

Kelly (2007): G. Kelly, "The Sphragis and Closure of the Res Gestae", in: J. Den Boeft et al. (eds.), Ammianus after Julian. The Reign of Valentinian and Valens in Books 26-31 of the Res Gestae, Leiden / Boston, 219-241.

Kelly (2008): G. Kelly, Ammianus Marcellinus. The Allusive Historian, Cambridge.

Matthews (1989): J. Matthews, The Roman Empire of Ammianus, London.

McCormack (1975): S. McCormack, “Latin Prose Panegyric”, in: T. A. Dorey (ed.), Empire and Aftermath. Silver Latin II, London / Boston, 143-205.

Momigliano (1980): A. Momigliano, "The Lonely Historian Ammianus Marcellinus", in: Id., Sesto contributo alla storia degli studi classici e del mondo antico, Roma, 143-157.

Nagel / Wood (2010): A. Nagel and Ch. Wood, Anachronic Renassaince, New York.

Nugent (1990): G. S. Nugent, “Ausonius',late antique poetics“ and ,post-modern“ literary theory”, Ramus 19, 26-50.

Pichon (1906): R. Pichon, Les derniers écrivains profanes, Paris.

Rees (2002): R. Rees, Layers of Loyalty in latin panegyric: AD 289-307, Oxford.

Rees (2012): R. Rees (ed.), Latin Panegyric, Oxford.

Rees (2013): R. Rees, “Pacatus the poet doing Plinian prose”, in: Gibson / Rees (2013) 241-259. Van Nuffelen (2012): P. Van Nuffelen, Orosius and the Rhetoric of History, Oxford.

Vessey (2015): M. Vessey, "Literature, Literary Histories, Latin Late Antiquity: The State of a Question”, in: J. Stenger (ed.), Spätantike Konzeptionen von Literatur, Heidelberg, 19-30. Wittchow (2001): F. Wittchow, Exemplarisches Erzählen bei Ammianus Marcellinus: Episode, Exemplum, Anekdote, München. 